\title{
Preface to the Focused Issue in Honor of Professor Philip Roe on the Occasion of His 80th Birthday
}

\author{
Rémi Abgrall ${ }^{1}$. Jennifer K. Ryan ${ }^{2} \cdot$ Chi-Wang Shu ${ }^{3}$ \\ Published online: 13 March 2020 \\ (c) Shanghai University 2020
}

On July 3-4, 2018, an international workshop was organised at the University of Cambridge, by Dr. Nikoforakis, to mark the 80th anniversary of a young scientist, Philip Roe.

Phil's scientific achievements are numerous, and it is quite difficult to adequately address his accomplishments in a short preface. Phil began his journey at Cambridge, first for his Bachelor's degree and then obtaining his Master's degree in 1962. He left for the Royal Aircraft Establishment (RAE) between 1962 to 1984 to work as an engineer. It was a period of adventure, and the design of mature CFD codes for solving the Euler equations was far from being achieved. Though the Lax-Wendroff theorem was known (published in 1960), as for many novelties, it had taken some time before it became a gold standard. At the RAE, Phil was assigned by his manager to work in a direction that eventually led to Roe's Riemann solver and flux. This method is now standard in every specialised textbook, but the jump from generalizing the Murman scheme for Euler equations to what is now commonly known as the Roe flux is far from obvious. Phil Roe relates this adventure, and others, in his "scientific autobiography", one of the papers of this focused issue.

Indeed, if one looks carefully at his celebrated 1981 paper, the method is not presented as a flux, but as a fluctuation method. In a nutshell, as any British sailor, Phil sits at the centre of his boat and tries to estimate where the wind is blowing from, so that he knows which information to forward to the sailors. The truly non-obvious point is to satisfy local conservation, and this is the precise role of the Roe linearization. It is only later, during his numerous stays at ICASE that, in the aim of explaining his method and thanks to many discussions with Bram van Leer and others, it was reinterpreted (a bit wrongly) as a numerical flux.

Rémi Abgrall

remi.abgrall@math.uzh.ch

Jennifer K. Ryan

jkryan@mines.edu

Chi-Wang Shu

chi-wang_shu@brown.edu

1 Institüt für Mathematik, Universität Zürich, Winterhurerstrasse 190, 8057 Zurich, Switzerland

2 Department of Applied Mathematics and Statistics, Colorado School of Mines, Golden, CO 80401, USA

3 Division of Applied Mathematics, Brown University, Providence, RI 02912, USA 
The management of the RAE quickly realised that it was an important discovery, and Phil was forbidden to publish his work for a few years. Although the "publish or perish" mentality was not as intense as it is nowadays, it still placed a difficult barrier in front of him. Nevertheless, Phil was recognised by the community, and in the 1980's he was often invited to the "Mecca" of CFD-ICASE at NASA Langley. He then left the RAE to become professor at Cranfield. Note that Phil has no $\mathrm{PhD}$, but a "relatively" good record of publications and inspiring concepts. This was certainly sufficient enough for the hiring committee at Cranfield. Then, in 1990, he was convinced by Bram van Leer to join the University of Michigan at Ann Arbor, where he remains.

Phil is still a young man. He has been and continues to be quite productive, in the genuine sense of the word: he produces ideas. He is certainly not prolific, in terms of the number of publications, but his work is and always has been inspiring. He has always been encouraging to junior researchers. Indeed, one of the authors of this preface remembers very well the day he received a small hand written letter from Professor Philip L. Roe from Cranfield. Phil was commenting on a paper that just appeared in some obscure journal: how could he have been so kind to get interested in this work, certainly important for the author, but was it enough?

Returning to Phil Roe's work, let us not forget to mention his work on limiters and TVD schemes, his work on fluctuation splitting schemes has inspired a generation of CFDers, around the von Karman Institute and beyond. More recently, he has fallen in love with a new concept, what he calls active flux. There is a whole community, in Germany and beyond, that are actively working on this new methodology. In all his work, there is at least one central concept: upwinding. Not only because this is important for a British sailor, but also because the enemy is dissipation.

To celebrate Phil, this workshop was organised in Cambridge, the university where everything started and where he still frequently visits. Some of the speakers have written articles that are contained in this focused issue.

Besides Phil's scientific autobiography, this issue contains several other articles, and all related in one way or another to Phil's work over the years. Christiane Helzel has written a paper on active flux. Louisa Michael and co-workers have written a paper on multiphysics applications where the method originates from Phil's work, long ago. Riccardo Demattè and co-workers have written a paper on ADER for hyperbolic equations with time reconstruction. Jun Zhu and Chi-Wang Shu have written a paper on a new class of WENO schemes and their behavior for steady problems. Daniel J. Frean and Jennifer K. Ryan have written a paper on the use of upwinding to optimise numerical dissipation and dispersion in DG schemes, inspired by discussions with Phil when he would visit Cambridge. Rémi Abgrall has written a paper on fluctuation splitting schemes. Phil also shares his views on the discretization of hyperbolic problems. Finally, Steven Jöns and Claus-Dieter Munz have written a paper about an approximate Riemann solver for advection-diffusion.

Thank you very much, Phil! 